Cite this: RSC Adv., 2014, 4, 15740

Accepted 18th March 2014

DOI: 10.1039/c4ra00348a

www.rsc.org/advances
Received 13th January 2014

\section{Melting temperatures deduced from molar volumes: a consequence of the combination of enthalpy/entropy compensation with linear cohesive free-energy densities $\uparrow$}

\begin{abstract}
Thibault Dutronc, ${ }^{\star}$ Emmanuel Terazzi* and Claude Piguet*
Enthalpy/entropy compensation is a general issue of intermolecular binding processes when the interaction between the partners can be roughly modelled with a single harmonic potential. Whereas linear H/S correlations are wished for by experimentalists, and often graphically justified, no inflexible law of thermodynamics supports the latter statement. On the contrary, the non-directional Ford's approach suggests logarithmic H/S relationships, which can be linearized only over narrow enthalpy/entropy ranges. Predictions covering larger domains require mathematical mapping obeying specific boundary conditions which are not compatible with linear plots. The analysis of solvent-free melting processes operating in six different classes of organic and inorganic materials shows that reciprocal Hill plots are acceptable functions for correlating melting enthalpies and entropies. The combination of H/S compensation with the observed linear dependence of the cohesive free energy densities with respect to the melting temperature eventually provides an unprecedented interdependence between melting temperatures and molar volumes. This procedure is exploited for the prediction of melting temperatures in substituted cyanobiphenyls.
\end{abstract}

\section{Introduction}

A rational control and programming of the various contributions to the changes in standard thermodynamic free energy $\Delta G_{\text {asso }}^{0}$, enthalpy $\Delta H_{\text {asso }}^{0}$ and entropy $\Delta S_{\text {asso }}^{0}$ of association accompanying the binding process depicted in eqn (1) is at the heart of self-assemblies occurring in biology, ${ }^{1}$ in physics ${ }^{2}$ and in chemistry $^{3}$ (|| refers to the activities of various partners).

$$
\mathrm{A}+\mathrm{B} \rightleftharpoons[\mathrm{AB}] \quad \Delta G_{\text {asso }}^{0}=\Delta H_{\text {asso }}^{0}-T \Delta S_{\text {asso }}^{0}=-R T \ln \left(\frac{|[\mathrm{AB}]|}{|\mathrm{A}||\mathrm{B}|}\right)
$$

Beyond the theoretically justified Gibbs free energy relationship (eqn (1), right-hand side), ${ }^{4}$ the simple modelling of the interaction operating between the partners in the $[\mathrm{AB}]$ pair by using a harmonic potential (i.e. a spring) suggests that the stronger the binding energy (measured as the potential well depth $u_{\text {min }} \propto \Delta H_{\text {asso }}^{0}$ ), the larger its force constant $\kappa$ (measured as its mean vibrational frequency $\nu=(1 / 2 \pi) \sqrt{\kappa / m_{\mathrm{r}}} \propto-\Delta S_{\text {asso }}^{0}$ where $m_{\mathrm{r}}$ is the reduced mass of the harmonic oscillator). ${ }^{5}$

Department of Inorganic and Analytical Chemistry, University of Geneva, 30 quai E. Ansermet, CH-1211 Geneva 4, Switzerland. E-mail: Claude.Piguet@unige.ch; Emmanuel.Terazzi@unige.ch; Thibault.Dutronc@unige.ch

$\dagger$ Electronic supplementary information (ESI) available. See DOI: 10.1039/c4ra00348a
Consequently, enthalpy and entropy changes are usually correlated for a simple intermolecular binding event with a propensity for compensation, i.e. $\Delta S_{\text {asso }}^{0}$ and $\Delta H_{\text {asso }}^{0}$ concomitantly decrease or increase. ${ }^{6}$ Recurrent, but empirical observations collected from series of association processes investigated in biology, in physics and in chemistry show that minor structural perturbations of the two partners lead to apparent linear correlations (eqn (2), left-hand side). The slope $\alpha$ of the $\Delta H_{i}$ versus $\Delta S_{i}$ plot has Kelvin units and is often referred to as the compensation temperature $T^{\mathrm{comp}}$, i.e. the temperature at which all association processes $i$ within a homogeneous series display the same compensation free energy change $\Delta G^{\text {comp }}=\gamma$ (eqn (2), center). Its re-writing at the right-hand side of eqn (2) is reminiscent of the Gibbs relationship, which may explain the rather common belief in the statement that linear enthalpy/ entropy compensation corresponds to a 'fourth law' of thermodynamics. ${ }^{6,7}$

$$
\begin{aligned}
\Delta H_{i}=\alpha \Delta S_{i}+\gamma= & T^{\mathrm{comp}} \Delta S_{i}+\Delta G^{\mathrm{comp}} \\
& \Rightarrow \Delta G^{\mathrm{comp}}=\Delta H_{i}-T^{\mathrm{comp}} \Delta S_{i}
\end{aligned}
$$

Theoretical approaches based on statistical thermodynamics include both attempts to refute the relevance of enthalpyentropy correlation, ${ }^{8}$ as well as to establish its signature as the result of (i) minor perturbations of equilibrium constants, ${ }^{9}$ (ii) partition functions governed by a Gaussian density of $\operatorname{states}^{6 \boldsymbol{b}}$ or (iii) underlying hidden thermodynamic processes. ${ }^{6 c}$ In 2005, 
(a)

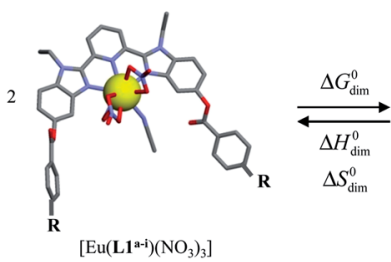

(b)

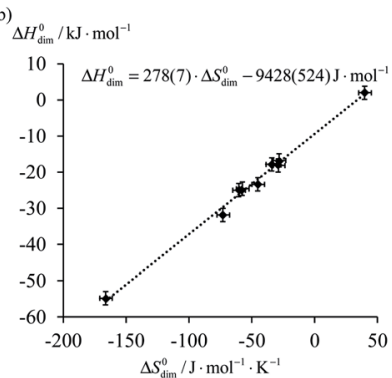

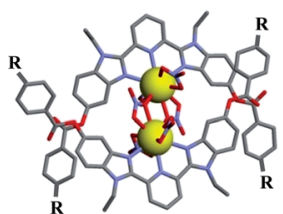

$\left[\mathrm{Eu}_{2}\left(\mathrm{L1}^{\mathrm{a}-\mathrm{i}}\right)_{2}\left(\mathrm{NO}_{3}\right)_{6}\right]$

(c)

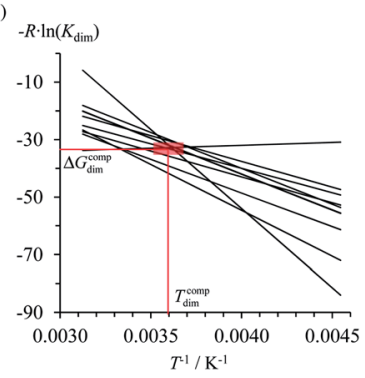

Fig. 1 (a) Schematic dimerization of complexes [Eu( $\left.\left.\mathrm{LI}^{a-i}\right)\left(\mathrm{NO}_{3}\right)_{3}\right]$ occurring in dichloromethane, (b) associated "linear" $\Delta H_{\text {dim }}^{0} v s . \Delta S_{\text {dim }}^{0}$ plots and (c) individual van't Hoff $\Delta G_{\text {dim }} / T$ vs. $T^{-1}$ plots for nine different $R$ substituents. ${ }^{13}$

Ford derived a non-quantum justification (see next section) claiming that enthalpy/entropy compensation $\left(T^{\text {comp }}>0\right.$ in eqn (2)) occurs when the minimum host-guest separation $r_{0}$ in the $[\mathrm{AB}]_{i}$ pairs remains constant within a series of intermolecular binding events. ${ }^{10}$ However, the linearity proposed in eqn (2) does not result from Ford's model, ${ }^{6 \boldsymbol{d}}$ and alternative physical justifications for parabolic ${ }^{5 a, 11}$ or rectangular-hyperbolic ${ }^{12}$ correlation for $H / S$ compensation have been proposed. In this context, Liu and Guo documented that the general emergence of linear $H / S$ compensation is often the consequence of statistical and mathematical artefacts arising from the data analysis. $^{6 \boldsymbol{a}}$ The dimerization process depicted in Fig. 1a illustrates this statement since the convincing linear relationship found for $H / S$ compensation (Fig. 1b) is significantly discredited when one considers the alternative, but equivalent van't Hoff plots which should cross at a common $\left\langle T^{\text {comp }} ; \Delta G^{\text {comp }}\right\rangle$ couple (red rectangle in Fig. 1c). ${ }^{13}$

Since complexation processes occurring in dilute solutions are complicated by unavoidable solvation changes, ${ }^{\mathbf{1 1}, \mathbf{1 4}}$ which may induce additional $H / S$ compensation phenomenon, ${ }^{15}$ we resorted to the melting of solids into isotropic liquid conducted in absence of solvents or of additives for getting a rough estimation of the strength of the intermolecular interactions occurring in a solid. ${ }^{16}$ These processes can be idealized as reversible $n^{\text {th }}$ order chemical reactions, in which $n$ identical monomeric units A associate into fully assembled entities $\mathrm{A}_{n}$ (eqn (3)). ${ }^{17}$

$$
n \mathrm{~A} \frac{\Delta G_{\text {asso }}^{0}=\Delta H_{\text {asso }}^{0}-T \Delta S_{\text {asso }}^{0}}{\Delta G_{\mathrm{m}}^{0}=\Delta H_{\mathrm{m}}^{0}-T \Delta S_{\mathrm{m}}^{0}} \mathrm{~A}_{n}
$$

At the melting temperature $T=T_{\mathrm{m}}$, the solid-liquid phase equilibrium implies that $\Delta G_{\text {asso }}=\Delta G_{\mathrm{m}}=0$ and $T_{\mathrm{m}}=\Delta H_{\mathrm{m}}^{0} / \Delta S_{\mathrm{m}}^{0}$ $=\Delta H_{\text {asso }}^{0} / \Delta S_{\text {asso }}^{0}=\Delta H_{\mathrm{m}} / \Delta S_{\mathrm{m}}$ when one reasonably assumes that the melting enthalpies $\Delta H_{\mathrm{m}}$ and entropies $\Delta S_{\mathrm{m}}$ operating at the melting temperatures are satisfyingly estimated by those
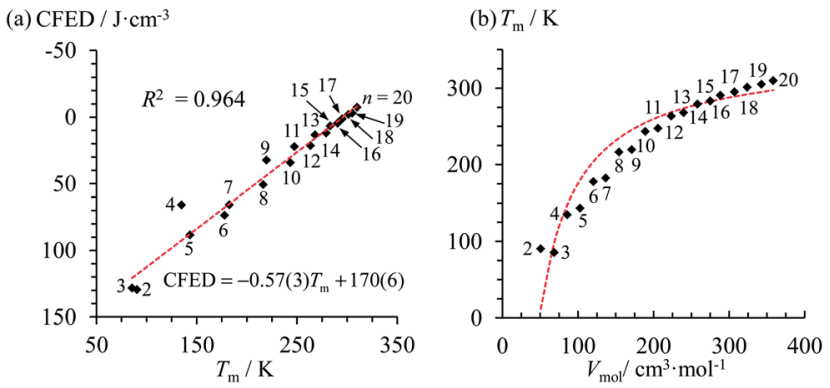

Fig. 2 Plots of (a) the cohesive free energy densities (CFED) versus the melting temperature $\left(T_{\mathrm{m}}\right)$ and (b) the melting temperature $\left(T_{\mathrm{m}}\right)$ versus the molar volumes $\left(V_{\text {mol }}\right)$ for saturated linear hydrocarbons $\mathrm{C}_{n} \mathrm{H}_{2 n+2}$ ( $n=2-20)$. The dotted red traces show the theoretical curves. Adapted from ref. 17

defined in the standard conditions (i.e. $\Delta H_{\mathrm{m}} \approx \Delta H_{\mathrm{m}}^{0}$ and $\Delta S_{\mathrm{m}} \approx$ $\left.\Delta S_{\mathrm{m}}^{0}\right) \cdot{ }^{17}$ Expressed at a common reference temperature $T^{0}$, each melting process is further characterized by its specific standard free energy change $\Delta G_{\mathrm{m}}^{0}=\Delta H_{\mathrm{m}}^{0}-T^{0} \Delta S_{\mathrm{m}}^{0} \approx \Delta H_{\mathrm{m}}-T^{0} \Delta S_{\mathrm{m}}$, which is further used to compute the standard cohesive free energy density CFED $=\Delta G_{\mathrm{m}}^{0} / V_{\text {mol }}\left(V_{\text {mol }}\right.$ is the molar volume $),{ }^{18} \mathrm{a}$ parameter estimating the average cohesive forces operating in the solid. ${ }^{19}$ Interestingly, CFED were found to be linearly correlated with the melting temperatures for series of association/dissociation processes obeying $H / S$ compensation (Fig. 2a), ${ }^{17}$ a phenomenon at the origin of the use of molar volumes for predicting the melting temperatures of linear alkanes (Fig. 2b). ${ }^{\mathbf{1 7}}$ However, the exclusive consideration of linear $H / S$ correlations limits this predictive model to the exploration of narrow domains of melting temperatures. ${ }^{17}$

\section{Theoretical background}

Following the formalism of molecular association proposed by Luo and Sharp, ${ }^{20}$ the equilibrium constant $K_{\text {asso }}^{\mathrm{A}, \mathrm{B}}$ associated with the simple binding event shown in eqn (1) can be written as eqn (4), where $c^{\theta}$ is the standard concentration of the reference state (fixed to $1 \mathrm{M}$ in this contribution), ${ }^{21} H(r, \Omega)$ is a bonding function depending on the separation $(r)$ and orientation $(\Omega)$ of the two partners in the $[\mathrm{AB}]$ pair $(H(r, \Omega)=1$ when the complex $[\mathrm{AB}]$ exists and $H(r, \Omega)=0$ otherwise), $\beta=\left(k_{\mathrm{b}} T\right)^{-1}$ stands for the thermal factor and $\omega(r, \Omega)$ is the potential mean force between $\mathrm{A}$ and $\mathrm{B.}^{10}$

$$
K_{\text {asso }}^{\mathrm{A}, \mathrm{B}}=\frac{|[\mathrm{AB}]|}{|\mathrm{A}||\mathrm{B}|}=\frac{c^{\theta}}{8 \pi^{2}} \int H(r, \Omega) \mathrm{e}^{-\beta \omega(r, \Omega)} \mathrm{d} r \mathrm{~d} \Omega
$$

Solving eqn (4) within the frame of the van't Hoff isotherm for a non-directional harmonic potential $\omega(r, \Omega)=u_{\min }^{\mathrm{A}, \mathrm{B}}+\left(\kappa^{\mathrm{A}, \mathrm{B}} / 2\right)$ $r^{2}$ operating between $\mathrm{A}$ and $\mathrm{B}$, whereby $u_{\text {min }}^{\mathrm{A}, \mathrm{B}}$ is the minimum potential energy and $\kappa^{\mathrm{A}, \mathrm{B}}$ is the force constant, leads to eqn (5) and (6), where $N_{\mathrm{Av}}$ is Avogadro number. ${ }^{10}$

$$
\Delta H_{\mathrm{asso}}^{\mathrm{A}, \mathrm{B}} / N_{\mathrm{Av}}=u_{\mathrm{min}}^{\mathrm{A}, \mathrm{B}}+\frac{3}{2} k_{\mathrm{b}} T
$$




$$
\Delta S_{\mathrm{asso}}^{\mathrm{A}, \mathrm{B}} / N_{\mathrm{Av}}=k_{\mathrm{b}} \ln \left[c^{\theta}\left(\frac{2 \pi e}{\beta \kappa^{\mathrm{A}, \mathrm{B}}}\right)^{3 / 2}\right]
$$

Interestingly, the enthalpy change $\Delta H_{\text {asso }}^{\mathrm{A}, \mathrm{B}}$ mainly depends on the magnitude of the interaction energy $u_{\text {min }}^{\mathrm{A}, \mathrm{B}}$, while the entropy change $\Delta S_{\text {asso }}^{\mathrm{A}, \mathrm{B}}$ is controlled by the force constant $\kappa^{\mathrm{A}, \mathrm{B}}$, the latter term being an estimation of the capacity of the bound system to gain residual degrees of freedom. The development of the harmonic potential at the minimum of a standard LennardJones potential $V_{\mathrm{L}-\mathrm{J}}$ shows that the absolute minimum energy of the attractive well depth corresponds to $u_{\text {min }}^{\mathrm{A}, \mathrm{B}}$ when the equilibrium $\mathrm{A} \cdots \mathrm{B}$ separation amounts to $2^{1 / 6} r^{0}\left(r^{0}\right.$ is the critical intermolecular $\mathrm{A} \cdots \mathrm{B}$ distance at which the interaction potential is zero: $V_{\mathrm{L}-\mathrm{J}}\left(r=r^{0}\right)=0$, see Fig. S1 in the ESI $\left.\dagger\right) .{ }^{6 d}$ Consequently, the total energy of the harmonic oscillator for the special motion amplitude $\left(2^{1 / 6} r^{0}-r^{0}\right)$ exactly corresponds to the well depth $u_{\text {min }}^{\mathrm{A}, \mathrm{B}}$ and eqn $(7)$ results. ${ }^{6 \boldsymbol{d}}$

$$
\begin{aligned}
-u_{\min }^{\mathrm{A}, \mathrm{B}} & =\frac{\kappa^{\mathrm{A}, \mathrm{B}}\left[r_{0}\left(1-2^{1 / 6}\right)\right]^{2}}{2} \Rightarrow \kappa^{\mathrm{A}, \mathrm{B}}=-\frac{2}{\left(1-2^{1 / 6}\right)^{2}\left(r_{0}\right)^{2}} u_{\min }^{\mathrm{A}, \mathrm{B}} \\
& =-f u_{\min }^{\mathrm{A}, \mathrm{B}}
\end{aligned}
$$

For a minor structural perturbation affecting a series of $\mathrm{A}$ and $\mathrm{B}$ partners, the minimum contact distance $r_{0}$ is constant within the resulting $[\mathrm{AB}]$ pairs and Ford's model (eqn (7), righthand side) predicts that the force constants $\kappa^{\mathrm{A}, \mathrm{B}}$ (which affects the entropy changes, eqn (6)) are linearly correlated with the potential well depths $u_{\text {min }}^{\mathrm{A}, \mathrm{B}}$ (which measures the enthalpy change, eqn (5)). Since the coefficient $f=2 /\left(1-2^{1 / 6}\right)^{2}\left(r_{0}\right)^{2}$ is positive, a larger cohesive energy between $\mathrm{A}$ and $\mathrm{B}$ in the [AB] pair (i.e. $u_{\text {min }}^{\mathrm{A}, \mathrm{B}}$ and $\Delta H_{\mathrm{asso}}^{\mathrm{A}, \mathrm{B}}$ become more negative) produces an increase in the force constant $\kappa^{\mathrm{A}, \mathrm{B}}$, hence in the mean vibrational frequency in the deeper potential. ${ }^{6 \boldsymbol{d}, 10}$ Since $\Delta S_{\text {asso }}^{\mathrm{A}, \mathrm{B}} / N_{\mathrm{Av}} \propto$ $-3 / 2 \ln \left[\kappa^{\mathrm{A}, \mathrm{B}}\right]$ in eqn (6), larger $\kappa^{\mathrm{A}, \mathrm{B}}$ induces more negative association entropies and $H / S$ compensation occurs. This model is nothing but the Einstein model for crystals, ${ }^{16,18 b, 22}$ from which Lindemann postulated that the melting of a solid occurs when the amplitude of the atomic thermal vibrations reaches some critical fraction of the equilibrium lattice spacing. ${ }^{23}$ Introducing eqn (7) into eqn (5) provides a simple correlation between the enthalpy change of the association process and the force constant in the bound state (eqn (8); $R=k_{\mathrm{b}} N_{\mathrm{Av}}$ is the ideal gas constant). Further introduction into eqn (6) gives the searched (logarithmic) dependence between $\Delta H_{\text {asso }}^{\mathrm{A}, \mathrm{B}}$ and $\Delta S_{\text {asso }}^{\mathrm{A}, \mathrm{B}}$ (eqn (9)). ${ }^{6 d}$

$$
\begin{gathered}
\frac{\Delta H_{\mathrm{asso}}^{\mathrm{A}, \mathrm{B}}}{N_{\mathrm{Av}}}=\frac{-\kappa^{\mathrm{A}, \mathrm{B}}}{f}+\frac{3}{2} k_{\mathrm{b}} T \Rightarrow \kappa^{\mathrm{A}, \mathrm{B}}=\frac{f}{N_{\mathrm{Av}}}\left(\frac{3}{2} R T-\Delta H_{\mathrm{asso}}^{\mathrm{A}, \mathrm{B}}\right) \\
\Delta S_{\mathrm{asso}}^{\mathrm{A}, \mathrm{B}}=R\left[\ln \left(c^{\theta}\right)+\frac{3}{2} \ln \left(\frac{2 \pi e R T}{f}\right)-\frac{3}{2} \ln \left(\frac{3}{2} R T-\Delta H_{\text {asso }}^{\mathrm{A}, \mathrm{B}}\right)\right]
\end{gathered}
$$

Its application to the melting of the $A_{n}$ assembly shown in eqn (3) requires $\Delta H_{\mathrm{m}}=-\Delta H_{\text {asso }}$ and $\Delta S_{\mathrm{m}}=-\Delta S_{\text {asso }}$, and eqn (10) eventually results

$$
\Delta S_{\mathrm{m}}=R\left[\frac{3}{2} \ln \left(\frac{3}{2} R T+\Delta H_{\mathrm{m}}\right)-\ln \left(c^{\theta}\right)-\frac{3}{2} \ln \left(\frac{2 \pi e R T}{f}\right)\right]
$$

Applied to the melting of a series of similar compounds, ${ }^{17}$ the force constant $\kappa^{\mathrm{A}, \mathrm{B}}$ can be approximated by a firstorder Taylor series around its average magnitude $\kappa_{0}^{\mathrm{A}, \mathrm{B}}$, and $\ln \left(\frac{3}{2} R T+\Delta H_{\mathrm{m}}\right)$ in eqn (10) can be replaced with $\ln \left(\frac{3}{2} R T+\Delta H_{\mathrm{m}, 0}\right)+\left(\Delta H_{\mathrm{m}}-\Delta H_{\mathrm{m}, 0}\right) /\left(\frac{3}{2} R T+\Delta H_{\mathrm{m}, 0}\right)$, where $\Delta H_{\mathrm{m}, 0}$ is the average melting enthalpy in the series. ${ }^{6 \boldsymbol{d}}$ However, the rapid divergence of the logarithmic function from a linear approximation restricts the use of linear $H / S$ compensation within this model to a narrow thermodynamic range of melting processes. In this contribution, we propose to improve the predictive capacity of the latter thermodynamic model via a thorough investigation of the melting processes occurring in metals, in various organic compounds and in inorganic oxides.

\section{Results and discussion}

\section{Modelling $H / S$ compensation}

Thanks to Yaws' efforts for gathering melting enthalpies and entropies (Fig. 3), ${ }^{24}$ we were able to select six families of compounds, linear alkanes (Table $\mathrm{S} 1 \dagger$ ), linear alkanoic acids (Table S2 $\dagger$ ), organosilanes (Table S3 $\dagger$ ), lanthanide metals (Table $\mathrm{S} 4 \dagger$ ), transition metals (Table $\mathrm{S} 5 \dagger$ ) and transition metal oxides (Table S6†), for which (i) the internal cohesion forces result from various types of intermolecular interactions (permanent and/or induced electric multipolar, H-bonds, covalency) and (ii) the melting temperatures span a broad domain. Assuming the justified approximation that $\Delta H_{\mathrm{m}}$ and $\Delta S_{\mathrm{m}}$ are essentially constant over a reasonable temperature range around a reference temperature $T^{0}$ taken as the average melting temperature within each series (Tables S1-S6 in the ESI $\dagger$ ), ${ }^{17}$ the experimental $\Delta S_{\mathrm{m}}$ versus $\Delta H_{\mathrm{m}}$ plots of the six individual series were fitted with Ford's model by tuning $f$ in eqn (10), but only a poor match

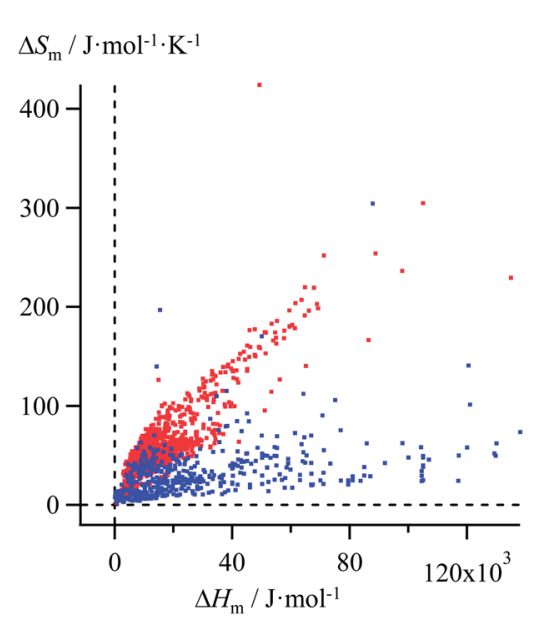

Fig. 3 Plot of melting entropies $\Delta S_{m}$ versus melting enthalpies $\Delta H_{m}$ for 764 randomly selected organic compounds (red markers) and 230 inorganic compounds (blue markers). ${ }^{24}$ 
(a) $\Delta S_{\mathrm{m}} / \mathrm{J} \cdot \mathrm{mol}^{-1} \cdot \mathrm{K}^{-1}$
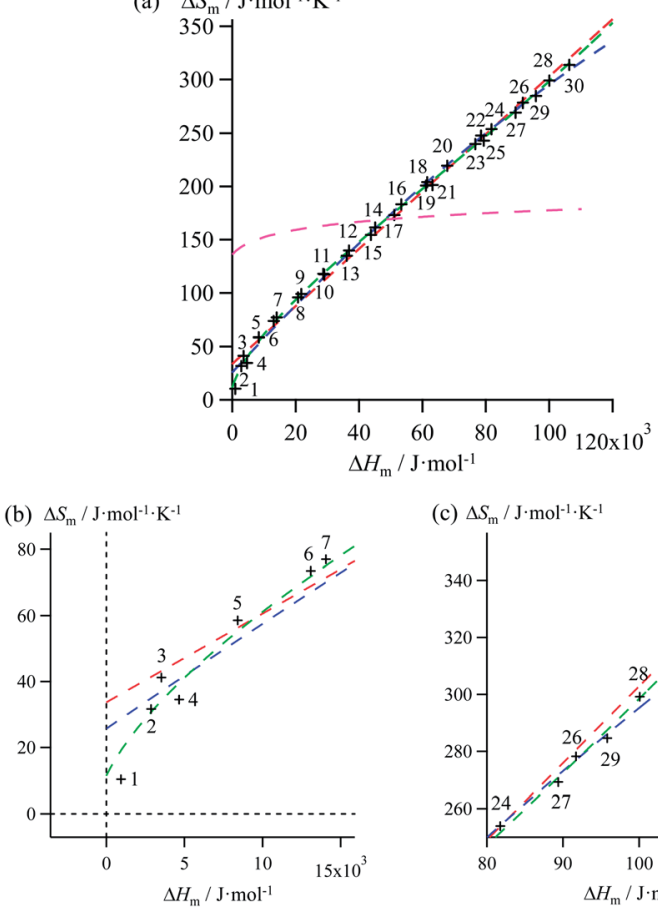

(c) $\Delta S_{\mathrm{m}} / \mathrm{J} \cdot \mathrm{mol}^{-1} \cdot \mathrm{K}^{-1}$

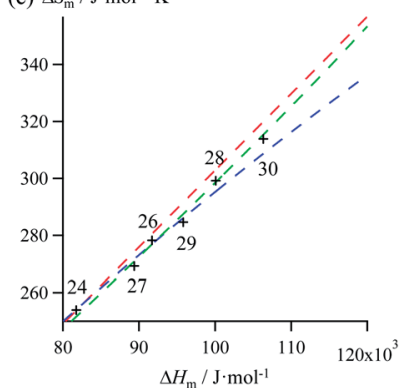

Fig. 4 (a) Full representation, (b) low enthalpy inset and (c) high enthalpy inset for plots of melting entropies $\Delta S_{m}$ versus melting enthalpies $\Delta H_{m}$ in linear alkanes $\mathrm{C}_{n} \mathrm{H}_{2 n+2}(n=1-30)$ and fitted unconstrained correlations using (i) Ford's approach (eqn (10), magenta trace), (ii) a linear H/S function (red trace), (iii) a parabolic H/S function (blue trace) or (iv) a reciprocal Hill plot (green trace).

results (magenta trace in Fig. 4 for linear alkanes and in Fig. S2S6 for the five other series in the ESI $\dagger$ ).

Mathematically speaking, the fitting process is logically improved by the stepwise consideration of additional tuneable parameters according to the following order: Ford's model (eqn (10), one tuneable parameter, magenta traces $)<$ linear fits $\left(\Delta S_{\mathrm{m}}=\right.$ $a+b \Delta H_{\mathrm{m}}$, two tuneable parameters, red traces $)<$ parabolic fits $\left(\Delta S_{\mathrm{m}}=a+b \Delta H_{\mathrm{m}}+c\left(\Delta H_{\mathrm{m}}\right)^{2}\right.$, three tuneable parameters, blue traces $)<$ reciprocal Hill fits $\left(\Delta S_{\mathrm{m}}=a\left[\left(\Delta H_{\mathrm{m}}-b\right) /\left(\Delta H_{\mathrm{m}}+c\right)\right]^{d}\right.$, four tuneable parameters, green traces; Fig. 4 and S2-S6 and Tables 1 and $\mathrm{S} 7-\mathrm{S} 11) \dagger$. The use of linear and parabolic fits is reminiscent of the use of first-order, respectively second-order Taylor polynomials for approaching eqn (10). ${ }^{17}$ Higher-order Taylor series are precluded by the well-known Runge's phenomenon, which strongly limits the approximation of logarithmic mapping by using power polynomials of increasing orders. ${ }^{25}$ On the contrary, the use of a Hill function has no mathematical justification for fitting logarithmic plots, but its stability over a large enthalpy range appeared to be a considerable advantage for fitting $H / S$ compensation (vide infra). A simple look at Fig. 3 indicates that $\Delta S_{\mathrm{m}} \rightarrow 0$ when $\Delta H_{\mathrm{m}} \rightarrow 0$ for melting processes recorded at normal pressure (in other words, the melting temperature $T_{\mathrm{m}}=$ $\Delta H_{\mathrm{m}} / \Delta S_{\mathrm{m}}$ does not diverge for $\left.\Delta S_{\mathrm{m}} \rightarrow 0\right),{ }^{26}$ a boundary condition which is not satisfied by the fitting traces collected in Fig. 4 and S2-S6 (ESI $\dagger$ ). Including the latter constraint into $H / S$ compensation results in the loss of one degree of freedom by fixing one parameter during the fitting process (Tables 1 and S7-S11, $\dagger$ column 1). Consequently, Ford's approach lacks of acceptable correlation (magenta traces in Fig. 5 and S7-S11, $\dagger$ no tuneable parameter), while linear approximations (red traces in Fig. 5 and $\mathrm{S} 7-\mathrm{S} 11, \dagger$ one tuneable parameter, $\left.\Delta S_{\mathrm{m}}=b \Delta H_{\mathrm{m}}\right)$ are dismissed because only a single melting temperature $T_{\mathrm{m}}=b^{-1}$ is tolerated along the complete series of compounds.

Therefore, among the four mathematical functions selected for extending the logarithmic $H / S$ dependence suggested by the simple Ford's model, only parabolic (blue traces, two tuneable parameters) and Hill (green traces, three tuneable parameters) plots provide satisfying fitted traces compatible with the inclusion of the extra boundary condition $\Delta S_{\mathrm{m}} \rightarrow 0$ when $\Delta H_{\mathrm{m}} \rightarrow 0$ (Fig. 5 and S7-S11, Tables 1 and S7-S11, ESI $\dagger$ ). ${ }^{6 a}$

\section{Cohesive free energy densities and three-dimensional $\Delta H_{\mathrm{m}}$, $\Delta S_{\mathrm{m}}, V_{\text {mol }}$ plots}

Taking Trouton's rule into account for the vaporization of liquids, ${ }^{27}$ Hildebrand introduced the concept of cohesion energy density $\mathrm{CED}=\left(\Delta H_{\mathrm{v}}-R T\right) / V_{\mathrm{mol}}$ for estimating the average cohesive forces operating within liquids and for predicting their vaporization temperatures $\left(\Delta H_{\mathrm{v}}\right.$ is the vaporization enthalpy). ${ }^{\mathbf{9}}$ In absence of valuable alternative to Trouton's rule fixing the melting entropy in solids, ${ }^{18}$ we have resorted to standard melting Gibbs free energy $\Delta G_{\mathrm{m}}^{0}=\Delta H_{\mathrm{m}}-T^{0} \Delta S_{\mathrm{m}}$ computed at a

Table 1 Entropy-enthalpy correlations fitted for linear alkanes $\mathrm{C}_{n} \mathrm{H}_{2 n+2}(n=1-30)^{a}$

\begin{tabular}{|c|c|c|c|c|c|}
\hline \multicolumn{6}{|l|}{ Unconstrained } \\
\hline Eqn (10) & $6(7) \times 10^{5}$ & - & - & - & - \\
\hline$\Delta S_{\mathrm{m}}=a+b \Delta H_{\mathrm{m}}+c\left(\Delta H_{\mathrm{m}}\right)^{2}$ & - & $26(2)$ & $3.2(1) \times 10^{-3}$ & $-5(1) \times 10^{-9}$ & - \\
\hline$\Delta S_{\mathrm{m}}=a\left[\left(\Delta H_{\mathrm{m}}-b\right) /\left(c-\Delta H_{\mathrm{m}}\right)\right]^{d}$ & - & $4.9(8) \times 10^{2}$ & $-7(8) \times 10^{2}$ & $3.3(5) \times 10^{5}$ & $6.2(3) \times 10^{-1}$ \\
\hline$\Delta S_{\mathrm{m}}=a+b \Delta H_{\mathrm{m}}$ & - & 0 & $3.17(6) \times 10^{-3}$ & - & - \\
\hline$\Delta S_{\mathrm{m}}=a+b \Delta H_{\mathrm{m}}+c\left(\Delta H_{\mathrm{m}}\right)^{2}$ & - & 0 & $4.2(2) \times 10^{-3}$ & $-1.3(2) \times 10^{-8}$ & - \\
\hline$\Delta S_{\mathrm{m}}=a\left[\left(\Delta H_{\mathrm{m}}-b\right) /\left(c-\Delta H_{\mathrm{m}}\right)\right]^{d}$ & - & $4.5(4) \times 10^{2}$ & 0 & $3.0(3) \times 10^{5}$ & $5.9(2) \times 10^{-1}$ \\
\hline
\end{tabular}

${ }^{a}$ Units used for polynomial fits: $a / \mathrm{J} \mathrm{mol}^{-1} \mathrm{~K}^{-1}, b / \mathrm{K}^{-1}$ and $c / \mathrm{J}^{-1} \mathrm{~mol} \mathrm{~K}^{-1}$. Units used for reciprocal Hill fits: $a / \mathrm{J} \mathrm{mol}{ }^{-1} \mathrm{~K}^{-1}, b$ and $c$ in $\mathrm{J} \mathrm{mol}^{-1}$. 

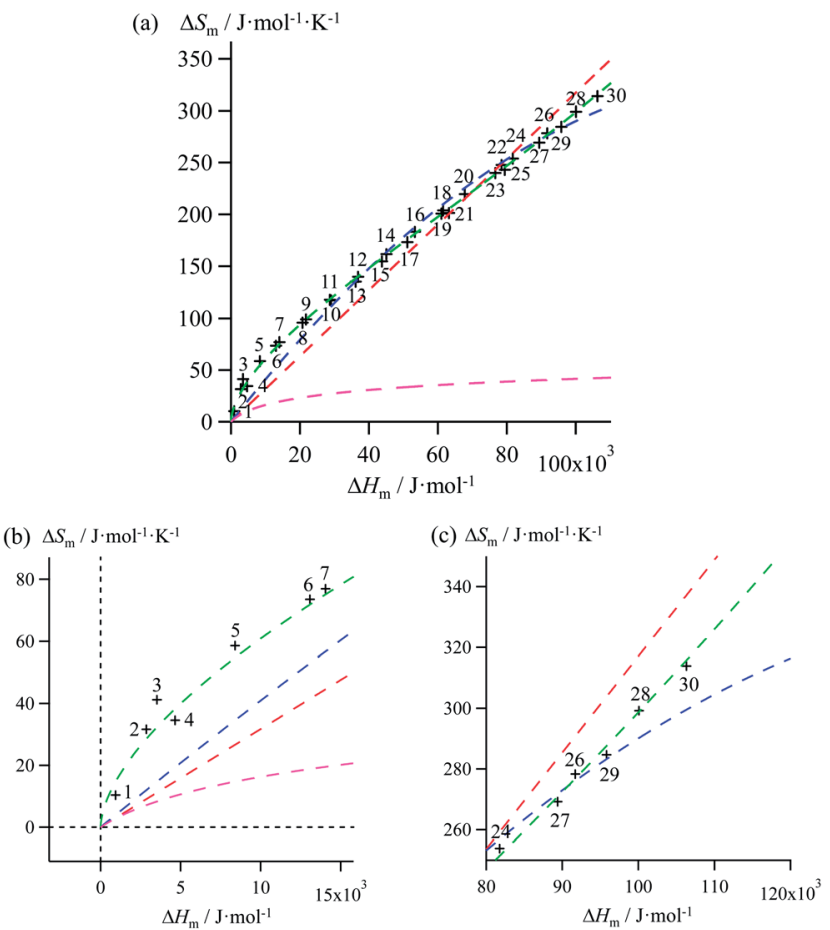

Fig. 5 (a) Full representation, (b) low enthalpy inset and (c) high enthalpy inset for plots of melting entropies $\Delta S_{m}$ versus melting enthalpies $\Delta H_{m}$ in linear alkanes $\mathrm{C}_{n} \mathrm{H}_{2 n+2}(n=1-30)$ and fitted constrained correlations including $\Delta S_{m} \rightarrow 0$ when $\Delta H_{m} \rightarrow 0$ and using (i) Ford's approach (eqn (10), magenta trace), (ii) a linear H/S function (red trace), (iii) a parabolic H/S function (blue trace) or (iv) a reciprocal Hill plot (green trace).

reference temperature $T^{0}$ for estimating the standard cohesive free energy densities CFED $=\Delta G_{\mathrm{m}}^{0} / V_{\mathrm{mol}}$ along each homogeneous series of compounds (Tables S1-S6, ESI $\dagger$ ). We are aware that the melting enthalpies $\left(\Delta H_{\mathrm{m}}\right)$ and entropies $\left(\Delta S_{\mathrm{m}}\right)$ are indeed collected at different temperatures for each compound (i.e. at $T_{\mathrm{m}}$ ) but their dependence on temperature is limited, ${ }^{17}$ and the choice of a reference temperature $T^{0}$ around the center of each series obeying $H / S$ compensation is thus acceptable for
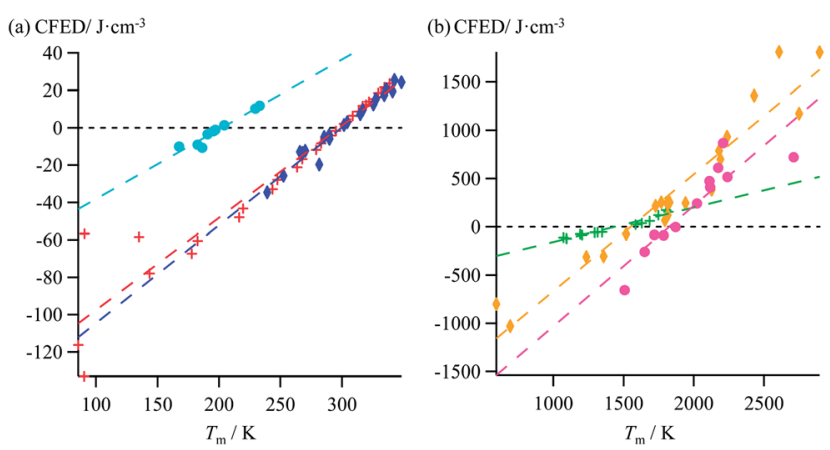

Fig. 6 Plots of standard cohesion free energy densities (CFED) versus the melting temperature $\left(T_{m}\right)$ for (a) linear alkanes (red trace), linear alkanoic acids (dark blue trace) and organosilanes (light blue trace), and (b) lanthanide metals (green trace), transition metals (orange trace) and transition metal oxides (magenta trace). computing $\Delta G_{\mathrm{m}}^{0}$. The experimental linear correlations observed between CFED and the associated melting temperatures $T_{\mathrm{m}}$ in Fig. 6 provide eqn (11), which interconnects $\Delta H_{\mathrm{m}}, \Delta S_{\mathrm{m}}$ and $V_{\mathrm{mol}}$ within each series (note that $T_{\mathrm{m}}=\Delta H_{\mathrm{m}} / \Delta S_{\mathrm{m}}$ )

$$
\mathrm{CFED}=\frac{\Delta G_{\mathrm{m}}^{0}}{V_{\mathrm{mol}}}=\frac{\Delta H_{\mathrm{m}}-T^{0} \Delta S_{\mathrm{m}}}{V_{\mathrm{mol}}}=\lambda T_{\mathrm{m}}+\mu
$$

Taken separately, eqn (11) is of limited interest for tuning melting temperatures by molecular design since only the molar volume $V_{\text {mol }}$ can be easily computed for unknown compounds, ${ }^{28}$ while satisfying estimations and/or modelling of melting

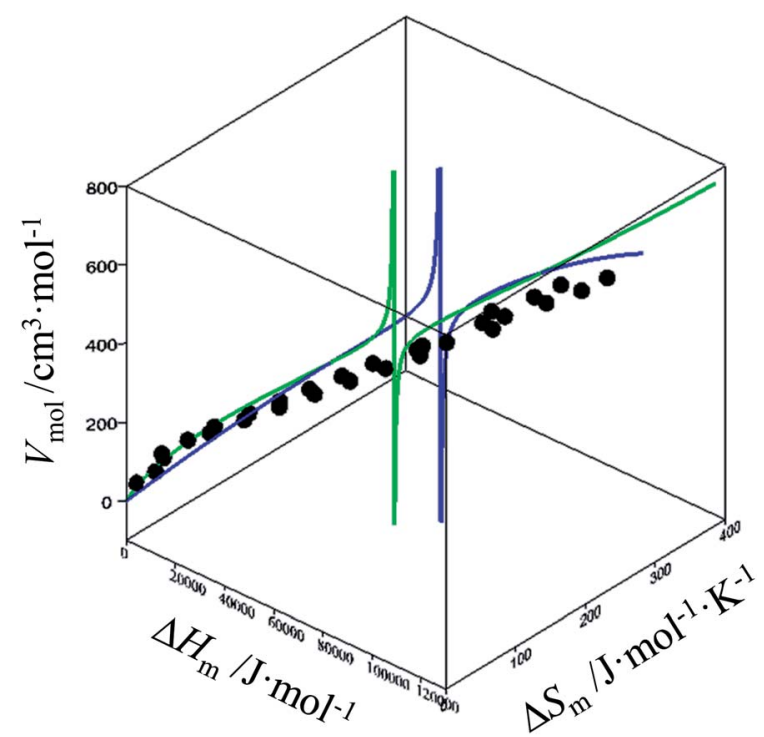

Fig. 7 Three-dimensional $V_{\text {mol }} \Delta H_{m}, \Delta S_{m}$ plot for linear alkanes fitted with a parabolic H/S function (blue trace) or a reciprocal Hill plots (green trace). An asymptotic behaviour is expected to occur when the melting temperature approaches the selected reference temperature $T^{0}=298.15 \mathrm{~K}$.

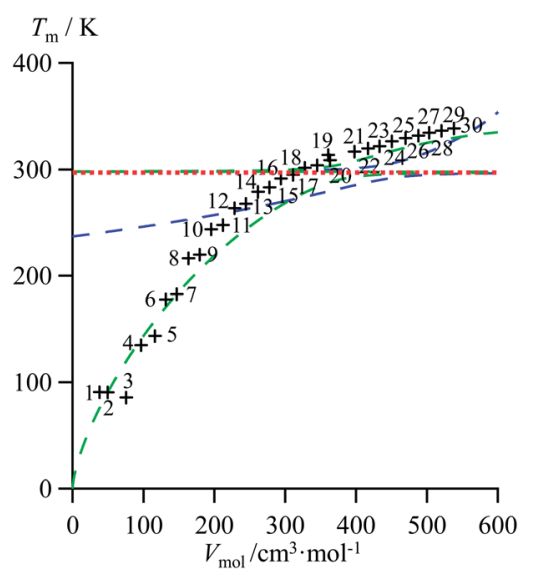

Fig. 8 Correlations between molar volumes $V_{\text {mol }}$ and melting temperatures $T_{m}$ for linear alkanes $\mathrm{C}_{n} \mathrm{H}_{2 n+2}(n=1-30)$ fitted using a parabolic H/S function (blue trace) or a reciprocal Hill plots (green trace). The horizontal dotted red trace corresponds to the asymptotical behaviour occurring when the melting temperature approaches the selected reference temperature $T^{0}=298.15 \mathrm{~K}$. 
entropies or enthalpies are difficult. ${ }^{29}$ However, the consideration of $H / S$ compensations operating in each series (Fig. 5 and S7-S11, ESI $\dagger$ ) provides a correlation $\Delta S_{\mathrm{m}}=g_{i}\left(\Delta H_{\mathrm{m}}\right)$ which can be introduced into eqn (11) to give eqn (12) when one reminds that $T_{\mathrm{m}}=\Delta H_{\mathrm{m}} / \Delta S_{\mathrm{m}}=\Delta H_{\mathrm{m}} / g_{i}\left(\Delta H_{\mathrm{m}}\right)\left(g_{i}\right.$ represents either a parabolic or a reciprocal Hill function).

$$
g_{i}\left(\Delta H_{\mathrm{m}}\right)\left[\Delta H_{\mathrm{m}}-T^{0} g_{i}\left(\Delta H_{\mathrm{m}}\right)-V_{\mathrm{mol}} \mu\right]-\lambda V_{\mathrm{mol}} \Delta H_{\mathrm{m}}=0
$$

For quadratic fits, $\Delta S_{\mathrm{m}}=g_{\text {quadra }}\left(\Delta H_{\mathrm{m}}\right)=b \Delta H_{\mathrm{m}}+c\left(\Delta H_{\mathrm{m}}\right)^{2}$ and eqn (12) corresponds to a polynomial, which can be solved to give an estimation of the melting enthalpy $\Delta H_{\mathrm{m}}$ as soon as the molar volumes $V_{\mathrm{mol}}$ of a target compound belonging to the series is at hand. The associated melting entropy $\Delta S_{\mathrm{m}}$ immediately results from $H / S$ correlation. The same procedure holds when using reciprocal Hill fits characterized by $\Delta S_{\mathrm{m}}=$ $g_{\mathrm{Hill}}\left(\Delta H_{\mathrm{m}}\right)=a\left[\Delta H_{\mathrm{m}} /\left(\Delta H_{\mathrm{m}}+c\right)\right]^{d}$. The quality of the correlations can be estimated in the three-dimensional $V_{\mathrm{mol}}, \Delta H_{\mathrm{m}}, \Delta S_{\mathrm{m}}$ plots for each series (Fig. 7 and S12-S16, ESI $\dagger$ ).

As an ultimate step, the combination of melting enthalpies and entropies into the melting temperatures $T_{\mathrm{m}}=\Delta H_{\mathrm{m}} / \Delta S_{\mathrm{m}}$ reduces the dimension of the correlation shown in Fig. 7 to give an easy-manageable $T_{\mathrm{m}}$ versus $V_{\mathrm{mol}}$ plot (Fig. 8 and S17-S21, ESI $\dagger$ ). The careful inspection of the latter two-dimensional plots indicate that, for any series under investigation, reciprocal Hill functions provide the best fits.

\section{Combination of $\mathrm{H} / \mathrm{S}$ compensation with linear cohesive free energy densities (CFED) for predicting the melting temperatures of substituted lipophilic cyanobiphenyls}

Lipophilic cyanobiphenyls with variable lengths ( $n$-CB) and substitutions $\left(12 \mathrm{Me}_{m}-\mathrm{CB}\right)$ have been considered as precursors for inducing liquid crystalline mesophases (Fig. 9a, Table S12, (a)

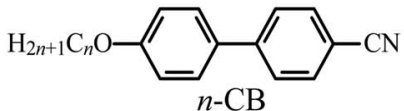

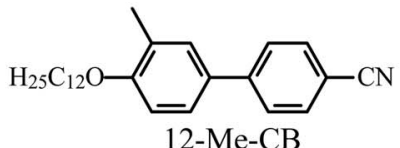<smiles>CCCCCOc1ccc(-c2ccc(C#N)cc2C)cc1C</smiles>

(b) $\Delta S_{\mathrm{m}} / \mathrm{J} \cdot \mathrm{mol}^{-1} \cdot \mathrm{K}^{-1}$

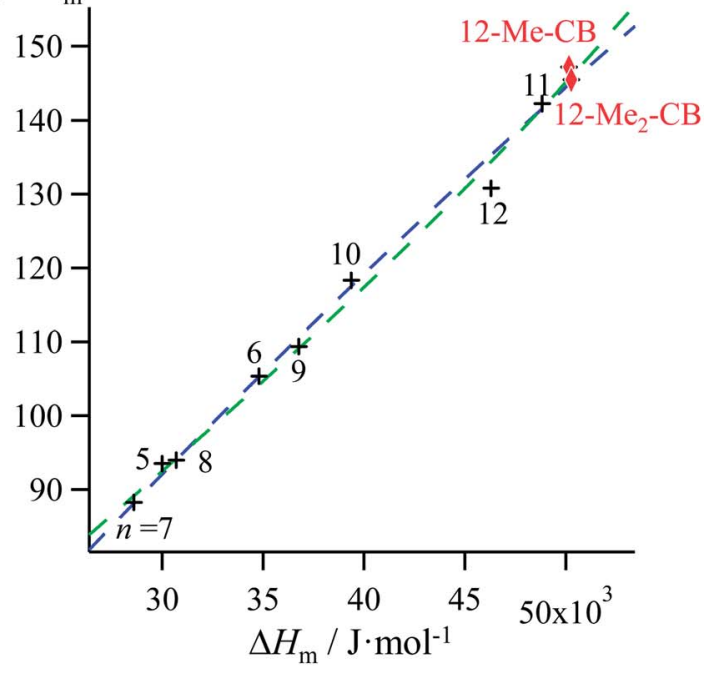

(d) (c) $\mathrm{CFED} / \mathrm{J} \cdot \mathrm{cm}^{-3}$

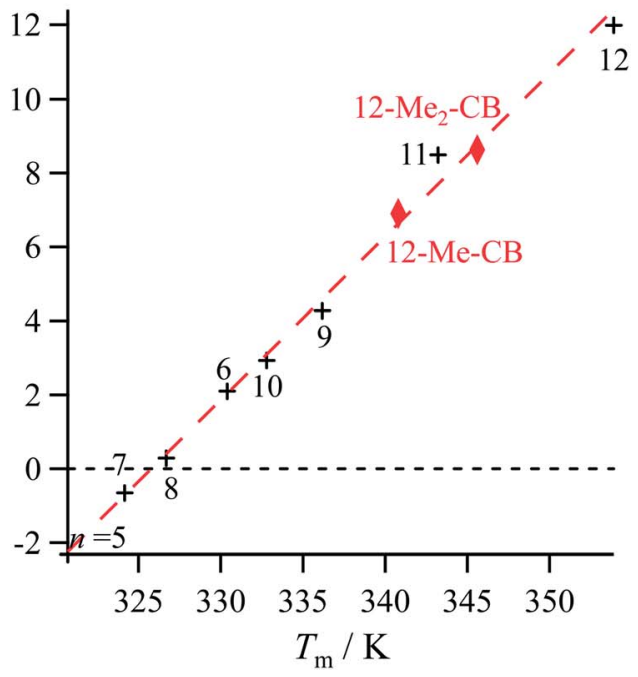

12

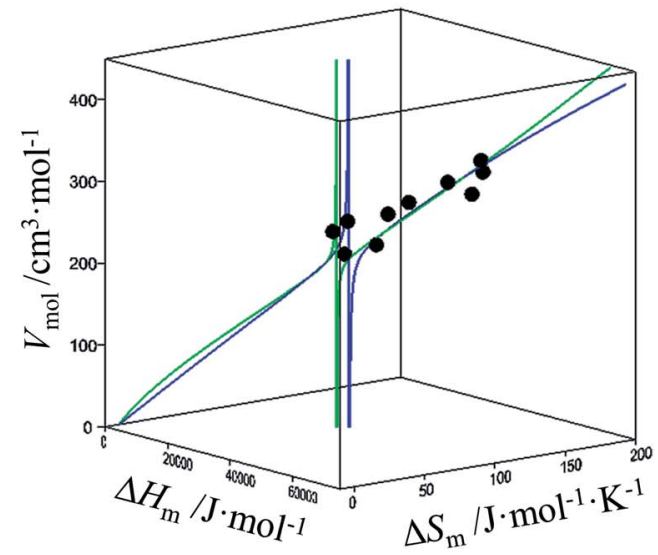

Fig. 9 (a) Chemical structures of substituted cyanobiphenyls with associated plots corresponding to (b) melting entropy versus melting enthalpy changes, (c) cohesion free energy densities (CFED) versus the melting temperature $\left(T_{\mathrm{m}}\right)$ and (d) three-dimensional $V_{\text {mol }}$, versus $\Delta H_{\mathrm{m}}$ and $\Delta S_{\mathrm{m}}$ fitted with a parabolic H/S function (blue trace) or a reciprocal Hill function (green trace). Reference temperature $T^{0}=325.92 \mathrm{~K}$. 


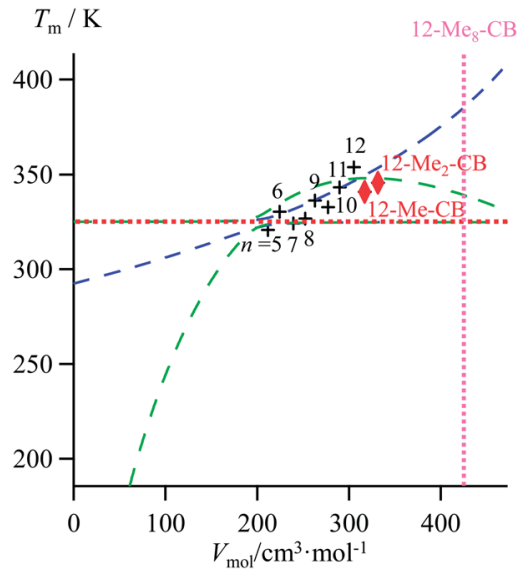

Fig. 10 Correlations between molar volumes $V_{\text {mol }}$ and melting temperatures $T_{m}$ for substituted lipophilic cyanobiphenyls fitted using a parabolic H/S function (blue trace) or a reciprocal Hill plots (green trace). The horizontal dotted red trace corresponds to the asymptotical behaviour occurring when the melting temperature approaches the selected reference temperature $T^{0}=325.95 \mathrm{~K}$. The vertical dotted magenta trace shows the predictions obtained for the permethylated cyanobiphenyl 12-Mes-CB.

ESI $\dagger) .{ }^{17}$ Both constrained quadratic and reciprocal Hill correlations satisfyingly model $H / S$ compensation along the cyanobiphenyl series (Fig. 9b), which further displays a linear CFED versus $T_{\mathrm{m}}$ plot according to eqn (11) (Fig. 9c). The threedimensional $V_{\mathrm{mol}}, \Delta H_{\mathrm{m}}, \Delta S_{\mathrm{m}}$ plot fitted with eqn (12) confirms the comparable quality of quadratic (blue trace) or Hill (green trace) functions (Fig. 9d). However, the trends predicted beyond the range of available experimental data significantly differ for the quadratic (blue trace) and Hill (green trace) plots, a feature highlighted in the reduced two-dimensional $T_{\mathrm{m}}$ versus $V_{\text {mol }}$ plot (Fig. 10). ${ }^{30}$

The ultimate step of our model offers the opportunity to predict the melting temperature for the unknown permethylated 4'-(dodecyloxy)-4-cyanobiphenyl compound (12$\mathrm{Me}_{8}-\mathrm{CB}$ ), for which a molecular volume of $688.5 \AA^{3}$, hence a molar volume of $V_{\mathrm{mol}}=N_{\mathrm{Av}} \times 688.5 \times 10^{-24}=414.2 \mathrm{~cm}^{3} \mathrm{~mol}^{-1}$ can be estimated. ${ }^{\mathbf{1 7}, 28}$ Depending on the choice of the fitting curve used for modelling $H / S$ compensation, an increase (quadratic fit) or a stagnation (Hill fit) of the melting temperature is expected, and one can therefore predicts $T_{\mathrm{m}}\left(12-\mathrm{Me}_{8}-\mathrm{CB}\right)$ $=380.5 \mathrm{~K}$ based on the quadratic fit and $340.7 \mathrm{~K}$ based on the Hill fit (Fig. 10). Since the experimental number of methyl groups bound to the cyanobiphenyl core in $12-\mathrm{Me}_{m}-\mathrm{CB}$ is currently limited to $m=2,{ }^{17}$ no definitive preference between quadratic and Hill predictions can be set. However, the stagnation of the melting temperature detected upon successive methylation $\left(T_{\mathrm{m}}(12-\mathrm{CB}) \geq T_{\mathrm{m}}(12-\mathrm{Me}-\mathrm{CB}) \approx T_{\mathrm{m}}\left(12-\mathrm{Me}_{2}-\mathrm{CB}\right)\right.$ in Fig. 10) has been confirmed by the systematic preparation of all the possible regioisomers of $12-\mathrm{Me}-\mathrm{CB}$ (4 isomers, average melting temperature $T_{\mathrm{m}}$ (monomethyl-CB) $=326(11) \mathrm{K}$ ), and those of 12- $\mathrm{Me}_{2}-\mathrm{CB}$ (4 isomers with one methyl group connected to each aromatic group, average melting temperature $T_{\mathrm{m}}$ $($ dimethyl-CB $)=327(16) \mathrm{K}) .{ }^{17}$ This trend represents a strong support in favor of the Hill plot as the best predictive tool within this novel series, in line with the previous analysis of linear alkane and alkanoic acids, organosilanes, lanthanide metals, transition metals and transition metal oxides.

\section{Conclusion}

The non-directional Ford's approach uses a single harmonic potential with a unique minimum contact distance for catching any intermolecular interaction. It predicts that, for a series of binding reactions involving structurally similar partners, the thermodynamic of association/dissociation is dominated by $H / S$ compensation and logarithmic $\Delta S$ versus $\Delta H$ dependences are expected. Applied to the melting processes occurring in six (arbitrarily selected) sets of chemical compounds, this rough model justifies the emergence of $H / S$ compensation, but it fails in reproducing accurate trends because the modelling of the intermolecular interactions operating in solid with the help of a single harmonic potential is a too big oversimplification. Increasing the number of degrees of freedom for approaching $H / S$ correlations restores satisfying fits when using linear or quadratic polynomials, or reciprocal Hill functions. The inclusion of the boundary condition $\Delta S_{\mathrm{m}} \rightarrow 0$ when $\Delta H_{\mathrm{m}} \rightarrow$ 0 recuses linear $H / S$ correlations and only quadratic or Hill plots are able to give satisfying $\Delta S_{\mathrm{m}}$ versus $\Delta H_{\mathrm{m}}$ fits. Standard empirical analysis of enthalpy/entropy compensation within the frame of this simple binding thermodynamics stops at this point, and no predictive tool is at hand for estimating the strength of intermolecular interactions and for programming melting temperatures. The additional concept of standard cohesive free energy density (CFED $=\Delta G_{\mathrm{m}}^{0} / V_{\mathrm{mol}}$ ) and its observed linear correlation with the melting temperatures (eqn (11)) introduces an unprecedented relationship between the molar volume, $V_{\mathrm{mol}}$, a parameter accessible to supramolecular chemists and the correlated melting enthalpies and entropies. For the six different series of compounds selected in this contribution, i.e. linear alkanes, linear alkanoic acids, organosilanes, lanthanide metals, transition metals and transition metal oxides, the ultimate reduced $T_{\mathrm{m}}$ versus $V_{\text {mol }}$ plots satisfyingly reproduce the experimental data. We notice that the use of reciprocal Hill plots provide the best fits for $H / S$ correlations in melting processes, and its application for rationalizing melting temperatures recently determined for substituted cyanobiphenyls provides reasonable predictions for currently unknown compounds in the series.

\section{Experimental}

The mathematical analyses were performed by using Igor Pro® (WaveMetrics Inc.) and Excel® (Microsoft) softwares.

\section{Acknowledgements}

Financial support from the Swiss National Science Foundation is gratefully acknowledged. 


\section{References and notes}

1 (a) M. Mammen, S.-K. Choi and G. M. Whitesides, Angew. Chem., Int. Ed., 1998, 37, 2754-2794; (b) A. Mulder, J. Huskens and D. N. Reinhoudt, Org. Biomol. Chem., 2004, 2, 3409-3424; (c) M. T. Rodgers and P. B. Armentrout, Acc. Chem. Res., 2004, 37, 989-998; V. M. Krishnamurthy, B. R. Bohall, V. Semetey and G. M. Whitesides, J. Am. Chem. Soc., 2006, 128, 5802-5812.

2 (a) L. Cademartiri, K. J. M. Bishop, P. W. Snyder and G. A. Ozin, Philos. Trans. R. Soc., A, 2012, 370, 2824-2847; (b) C.-A. Palma, M. Cecchini and P. Samori, Chem. Soc. Rev., 2012, 41, 3713-3730; (c) J. Li, P. Nowak and O. Sijbren, J. Am. Chem. Soc., 2013, 135, 9222-9239.

3 (a) J. D. Badjic, A. Nelson, S. J. Cantrill, W. B. Turnbull and J. F. Stoddart, Acc. Chem. Res., 2005, 38, 723-732; (b) H.-J. Schneider, Angew. Chem., Int. Ed., 2009, 48, 39243977; (c) C. Piguet, Chem. Commun., 2010, 46, 6209-6231; (d) R. Chakrabarty, P. S. Mukherjee and P. J. Stang, Chem. Rev., 2011, 111, 6810-6918.

4 T. Engel and P. Reid, Physical Chemistry, Pearson Benjamin Cummings, San Francisco, 2006, pp. 113-122.

5 (a) M. S. Searle, M. S. Westwell and D. H. Williams, J. Chem. Soc., Perkin Trans. 2, 1995, 141-151; (b) C. Neal, A. Starace and M. Jarrold, Phys. Rev. B: Condens. Matter Mater. Phys., 2007, 76, 054113.

6 (a) L. Liu and Q.-X. Guo, Chem. Rev., 2001, 101, 673-695; (b) K. Sharp, Protein Sci., 2001, 10, 661-667; (c) E. B. Starikov and B. Norden, J. Phys. Chem. B, 2007, 111, 14431-14435; (d) C. Piguet, Dalton Trans., 2011, 40, 8059-8071.

7 (a) J. E. Leffler, J. Org. Chem., 1955, 20, 1202-1231; (b) D. H. Williams, E. Stephens, D. P. O'Brien and M. Zhou, Angew. Chem., Int. Ed., 2004, 43, 6596-6616; (c) D. H. Leung, R. G. Bergman and K. N. Raymond, J. Am. Chem. Soc., 2008, 130, 2798-2805.

8 (a) R. Lumry, Methods Enzymol., 1995, 259, 628-720; (b) A. Cornish-Bowden, J. Biosci., 2002, 27, 121-126.

9 K. F. Freed, J. Phys. Chem. B, 2011, 115, 1689-1692.

10 (a) D. M. Ford, J. Am. Chem. Soc., 2005, 127, 16167-16170; (b) D. M. Ford, Adsorption, 2005, 11, 271-277.

11 V. S. Shaikh, S. S. Terdale, A. Ahamad, G. R. Gupta, D. H. Dagade, D. G. Hundiwale and K. J. Patil, J. Phys. Chem. B, 2013, 117, 16249-16259.

12 E. B. Starikov and B. Norden, J. Phys. Chem. B, 2009, 113, 4698-4707.

13 T. B. Jensen, E. Terazzi, K. Buchwalder, L. Guénée, H. Nozary, K. Schenk, B. Heinrich, B. Donnio, D. Guillon and C. Piguet, Inorg. Chem., 2010, 49, 8601-8619.

14 (a) B. M. Castellano and D. K. Eggers, J. Phys. Chem. B, 2013, 117, 8180-8188; (b) E. Terazzi, A. Zaïm, B. Bocquet, J. Varin, L. Guénée, T. Dutronc, J.-F. Lemonnier, S. Floquet, E. Cadot, B. Heinrich, B. Donnio and C. Piguet, Eur. J. Inorg. Chem., 2013, 3323-3333.

15 (a) E. Fisicaro, C. Compari and A. Braibanti, Phys. Chem. Chem. Phys., 2004, 6, 4156-4166; (b) J. M. Ward, N. M. Gorenstein, J. Tian, S. F. Martin and C. B. Post,
J. Am. Chem. Soc., 2010, 132, 11058-11070; (c) B. Breiten, M. R. Lockett, W. Sherman, S. Fujita, M. Al-Sayah, H. Lange, C. M. Bowers, A. Heroux, G. Krilov and G. M. Whitesides, J. Am. Chem. Soc., 2013, 135, 1557915584.

16 S. Zamith, F. Chirot and J.-M. L'Hermite, Europhys. Lett., 2010, 92, 13004.

17 T. Dutronc, E. Terazzi, L. Guénée, K.-L. Buchwalder, A. Spoerri, D. Emery, J. Mareda, S. Floquet and C. Piguet, Chem.-Eur. J., 2013, 19, 8447-8456.

18 Since Richard's rule only holds for metals, the entropies of melting for organic compounds cannot be taken as constant, and the CFED parameter is thus derived from the free energy change (a) G. P. Tiwari, Met. Sci., 1978, 12, 317-320; (b) M. E. Glicksman, Principle of Solidification, Springer, New York, 2011, p. 22.

19 (a) M. Dunkel, J. Phys. Chem., 1928, A138, 42-54; (b) J. H. Hildebrand and R. L. Scott, The Solubility of Nonelectrolytes, Reinhold Pub Group, New York, 3rd edn, 1950; (c) R. F. Fedors, Polym. Eng. Sci., 1974, 14, 147-154; (d) A. F. M. Barton, Handbook of Solubility Parameters and Other Cohesion Parameters, CRC Press, 2nd edn, 1991, p. 127.

20 H. B. Luo and K. Sharp, Proc. Natl. Acad. Sci. U. S. A., 2002, 99, 10399-10404.

21 D. Munro, Chem. Br., 1977, 13, 100-105.

22 (a) A. Einstein, Ann. Phys., 1907, 22, 180-190; (b) D. W. Rogers, Einstein's other Theory, the Plank-BoseEinstein Theory of Heat Capacity, Princeton University Press, New Jersey, 2005.

23 F. A. Lindemann, Phys. Z., 1910, 11, 609-612.

24 C. L. Yaws, Yaws Handbook of Thermodynamics and Physical Properties of Chemical Compounds, Knovel-Elsevier, Norwich New York, 2003.

25 C. Runge, Zeitschrift für Mathematik und Physik, 1901, 46, 224-243.

26 Under critical conditions of pressure, a continuous transition from crystal to melt displaying $\Delta S_{\mathrm{m}}=0$ and $\Delta V_{\mathrm{m}}=0$ whilst $\Delta H_{\mathrm{m}} \neq 0$ is theoretically justified. However, no experimental demonstration of this prediction is currently available because of the extreme pressure conditions required for the most promising candidatesA. R. Ubbelohde, Melting and Crystal Structures, Clarendon Press, Oxford, 1965, pp. 19-24.

27 Trouton's rule states that the entropy of vaporization for any liquid is almost the same value, about $85-88 \mathrm{~J} \mathrm{~K}^{-1} \mathrm{~mol}^{-1}$. Consequently, the vaporization temperature entirely depends on the vaporization enthalpy. F. T. Trouton, London, Edinburgh Dublin Philos. Mag. J. Sci., 1884, 18, 54-57.

28 The molar volumes $V_{\text {mol }}=N_{\mathrm{Av}} \times V_{\text {Connolly }} \times 10^{-24}$ in $\mathrm{cm}^{3}$ $\mathrm{mol}^{-1}$ are deduced from the Connollyvolumes expressed in $\AA^{3}$. $V_{\text {Connolly }}$ are obtained from the building of the Connolly surfaces around the molecular structures of the compounds observed in their crystal structures (when available) or in their gas-phase structures modelled by semi-empirical PM3 method (a) M. L. Connolly, Science, 1983, 221, 709-713; (b) M. L. Connolly, J. Appl. Crystallogr., 
1983, 16, 548-558; (c) J. J. P. Stewart, J. Comput. Chem., 1989, 10, 209-220; (d) J. J. P. Stewart, J. Comput. Chem., 1989, 10, 221-264.

29 (a) A. R. Katritzky, U. Maran, M. Karelson and V. S. Lobanov, J. Chem. Inf. Comput. Sci., 1997, 37, 913-919, and references therein; (b) Y. Tsuchiya, H. Hasegawa and T. Iwatsubo, J. Chem. Phys., 2001, 114, 2484-2488; (c) Y. Tsuchiya,
H. Hasegawa and T. Iwatsubo, Jpn. J. Appl. Phys., Part 1, 2003, 42, 6508-6511.

30 For any cyanobiphenyl located close to the asymptote (i.e. possessing molar volumes in the $200-260 \mathrm{~cm}^{3} \mathrm{~mol}^{-1}$ range), a simple change of the reference temperature provides its unambiguous location on either part of the fitting curve (Fig. S22, ESI $\dagger$ ). 